\title{
Natural geometric unit system and electron magnetic moment anomaly
}

\author{
JANUSZ J. “JANI” KOWALSKI ${ }^{1(\mathrm{a})}$
}

${ }^{1}$ Carrer dels Serrans 8D, Platja de la Pobla de Farnals, Spain

\begin{abstract}
Consequences of implementation of the natural geometric unit system (the SG) based on the pre-2019 SI system, in which four fundamental physical constants undergo joint numerical and dimensional normalization to unity $\mathrm{c}=\mathrm{G}=\mathrm{k}=\mathrm{h}=$ 1 , with only one base geometric unit $\mathbf{u}$ equal to $\eta \mathrm{h} \cdot \mathrm{G} / \mathrm{c}^{3} \mid \mathrm{m}$, where the Newtonian gravitational constant $\mathbf{G} \approx \mathbf{6 . 6 7 3} 655205 \cdot$ $10^{-11} \mathbf{m}^{3} /\left(\mathbf{k g} \cdot \mathbf{s}^{2}\right)$, are further explored. In addition to the earlier hypothesized simple electron mass to charge ratio formula $\mathbf{m}_{\mathrm{e}}=$ $\mathrm{e} /\left(2^{9} \pi \alpha\right)$, and formulas for stable quarks rest masses: quark $\mathbf{u ~ m}_{\mathbf{u}}=\sqrt{(2 / 3)} /\left(\mathbf{2}^{7} \pi \mathbf{V}(\pi \alpha)\right) \mathbf{u}$, equivalent of $2.360 \mathrm{MeV} / \mathrm{c}^{2}$ and quark $\mathrm{d} \mathbf{m}_{\mathbf{d}}=\sqrt{(1 / 3)^{-1}} /\left(2^{7} \pi \sqrt{ }(\pi \alpha)\right) \mathbf{u}$, equivalent of $5.007 \mathrm{MeV} / \mathrm{c}^{2}$, a simple formula for electron magnetic moment anomaly is proposed $\alpha / 2 \pi-(\alpha / 2 \pi)^{2}-2^{8}(\alpha / 2 \pi)^{3}-2^{12}(\alpha / 2 \pi)^{4}-2^{16}(\alpha / 2 \pi)^{5}-2^{24}(\alpha / 2 \pi)^{6} \approx 0.001159652180$. The finding supports the research area of purely geometric modelling of the fundamental physical forces and their unification. It seems plausible, that in the SG, with use of half integer powers of $2,3, \pi$ and $\alpha$ only, all the fundamental properties of stable matter and electromagnetic radiation could be described.
\end{abstract}

Introduction. - Let us define the natural geometric unit system as in [1]

The 'natural geometric unit system' (in short SG) is the SI system [2] with the pre-2019 magnetic constant definition [3] $\mu_{0}=4 \pi \mathrm{k} / \mathrm{c}^{2}=4 \pi / 10^{7}\left[\mathrm{~kg} \cdot \mathrm{m} / \mathrm{C}^{2}\right]$, in which we set constants $\mathbf{c}=\mathbf{G}=\mathbf{k}=\mathbf{h}=\mathbf{1}$ and define a base unit $\mathbf{u}=\mathbf{V}\left|\mathbf{h} \cdot \mathbf{G} / \mathrm{c}^{3}\right| \mathbf{m}$. It is equivalent to adding the following dependencies between units that are assumed to be independent in the unmodified SI:

$$
\begin{aligned}
& \text { a. } \quad \mathbf{s}=\mathbf{v}\left|\mathbf{c}^{5} /(\mathbf{h G})\right| \mathbf{u}, \mathbf{m}=\mathbf{v}\left|\mathbf{c}^{3} /(\mathbf{h G})\right| \mathbf{u} \\
& \text { b. } \mathbf{k g}=\mathbf{v}|\mathbf{G} /(\mathbf{h c})| \mathbf{u}, \\
& \text { c. } \quad \mathrm{C}=\mathbf{v}|\mathbf{c} / \mathbf{h}| \mathbf{V} 10^{-7} \mathbf{u},
\end{aligned}
$$

where $|\mathrm{x}|$ denotes the dimensionless, numerical part of $\mathrm{x}$.

The symbols denote: $\quad \mathrm{c}$ - the speed of light in vacuum,
$\mathrm{G}$ - the Newtonian gravitational constant,
$\mathrm{k}$ - the Coulomb constant,
$\mathrm{h}$ - the Planck constant.

Consistency of conversion of values of physical properties from SI to SG is preserved when $\mathrm{m}^{3} /\left(\mathrm{kg} \cdot \mathrm{s}^{2}\right)=\mathrm{kg} / \mathrm{C} \cdot \nabla\left|\mathrm{k} / \mathrm{G}^{3}\right|$, the relation being a direct implication of the SG definition.

Let us postulate, that electron rest mass $\mathbf{m}_{\mathrm{e}}$ is bound to elementary charge $\mathbf{e}$ by the formula in the SI $\mathbf{m}_{\mathrm{e}}=|\mathbf{G}| \mathbf{k g} / \mathbf{C ~ e / ( 2 ^ { 9 } \pi \alpha )}$ and by the formula in the $S G \mathbf{m}_{\mathrm{e}}=\mathrm{e} /\left(2^{9} \pi \alpha\right)$, where $\alpha$ is the fine structure constant and $\mathbf{G} \approx \mathbf{6 . 6 7 3} 655205 \cdot 10^{-11} \mathrm{~m}^{3} /\left(\mathbf{k g} \cdot \mathrm{s}^{2}\right)$. Arguments for consistency of the postulated value of $\mathrm{G}$ with current state of research on the subject are given in [1].

(a) E-mail: kowalski@message.pl 
Electron magnetic moment anomaly. - With the definition and the postulate proposed in the introduction and with currently defined in the SI as exact the values of $\mathrm{c}=2.99792458 \cdot 10^{8} \mathrm{~m} / \mathrm{s}, \mathrm{h}=6.62607015 \cdot 10^{-34} \mathrm{~J} \cdot \mathrm{s}$ and $\mathrm{e}=1.602176634$ $\cdot 10^{-19} \mathrm{C}$; and calculated value of $\alpha \approx 7.297352569 \cdot 10^{-3}$ (in the SI $\alpha=\mathrm{e}^{2} 2 \pi / \mathrm{h} \cdot \mathrm{k} / \mathrm{c}$, in the SG $\alpha=\mathrm{e}^{2} 2 \pi$ ) we can formulate the following table of the fundamental electron properties with values consistent with CODATA 2018 [5]

Table 1: The fundamental electron properties.

\begin{tabular}{|c|c|c|c|c|c|}
\hline Property & Formula in SI & $\begin{array}{l}\text { Value in SI } \\
\text { (grey = exact value) }\end{array}$ & $\begin{array}{l}\text { Conversion to SG unit } \\
\text { (all values }=1 \text { ) }\end{array}$ & Value in SG & Formula in SG \\
\hline $\begin{array}{l}\text { electron Compton } \\
\text { wavelength }\end{array}$ & $\begin{array}{l}\lambda_{\mathrm{C}}=1 / \mathrm{c} \cdot 2^{9} \pi /(|\mathrm{G}| \mathrm{kg} / \mathrm{C}) \\
\cdot \sqrt{ }(\mathrm{h} \cdot 2 \pi \alpha \cdot \mathrm{k} / \mathrm{c})\end{array}$ & $2.426310239 \cdot 10^{-12} \mathrm{~m}$ & $\begin{array}{l}\sqrt{ }\left|\mathrm{G}^{3} / \mathrm{k}\right| \cdot \\
\sqrt{ }\left|\mathrm{c}^{3} /(\mathrm{hG})\right| \mathrm{u} / \mathrm{m}= \\
|\mathrm{G}| \mathrm{\eta}|\mathrm{c} / \mathrm{h}| \sqrt{ } 10^{7} \mathrm{u} / \mathrm{m}\end{array}$ & $3.444232279 \cdot 10^{2} \mathrm{u}$ & $\lambda_{C}=2^{9} \pi \sqrt{ }(2 \pi \alpha)$ \\
\hline $\begin{array}{l}\text { double magnetic flux } \\
\text { quantum }\end{array}$ & $2 \Phi_{0}=\sqrt{ }(\mathrm{h} \cdot 2 \pi / \alpha \cdot \mathrm{k} / \mathrm{c})$ & $4.135667696 \cdot 10^{-15} \mathrm{~Wb}$ & $\nabla|1 /(\mathrm{hc})| \sqrt{ } 10^{7} \mathrm{u} / \mathrm{Wb}$ & $2.934318620 \cdot 10 \mathrm{u}$ & $2 \Phi_{0}=\sqrt{ }(2 \pi / \alpha)$ \\
\hline Planck length $\cdot \sqrt{ }(2 \pi)$ & $1_{p}=\sqrt{ }\left(h \cdot G / c^{3}\right)$ & $4.051154841 \cdot 10^{-35} \mathrm{~m}$ & $\eta\left|c^{3} /(h G)\right| u / m$ & $1 \mathrm{u}$ & $\mathrm{u}$ \\
\hline elementary charge & $\mathrm{e}=\sqrt{ }(\mathrm{h} \cdot \alpha /(2 \pi) \cdot \mathrm{c} / \mathrm{k})$ & $1.602176634 \cdot 10^{-19} \mathrm{C}$ & $\sqrt{ }|\mathrm{c} / \mathrm{h}| \sqrt{ } 10^{-7} \mathrm{u} / \mathrm{C}$ & $3.407946203 \cdot 10^{-2} \mathrm{u}$ & $\mathrm{e}=\sqrt{ }(\alpha /(2 \pi))$ \\
\hline electron rest mass & $\begin{array}{l}\mathrm{m}_{\mathrm{e}}=|\mathrm{G}| \mathrm{kg} / \mathrm{C} /\left(2^{9} \pi\right) \\
\cdot \sqrt{ }(\mathrm{h} /(2 \pi \alpha) \cdot \mathrm{c} / \mathrm{k})\end{array}$ & $9.109383702 \cdot 10^{-31} \mathrm{~kg}$ & $\begin{array}{l}\eta \mathrm{k} / \mathrm{G}^{3} \mid \\
\eta \mathrm{G} /(\mathrm{hc}) \mid \mathrm{u} / \mathrm{kg}= \\
1 /|\mathrm{G}| \mathrm{\eta}|\mathrm{c} / \mathrm{h}| \sqrt{ } 10^{-7} \mathrm{u} / \mathrm{kg}\end{array}$ & $2.903404646 \cdot 10^{-3} \mathrm{u}$ & $\mathrm{m}_{\mathrm{e}}=1 /\left(2^{9} \pi \sqrt{ }(2 \pi \alpha)\right)$ \\
\hline
\end{tabular}

Conceptualizing Aristotle's approach to space (spacetime) and matter, which he also attributed rightfully to Plato as argued by J. Fritsche in [4]: "In Physics IV:2, Aristotle argues for private Space of a body as its form (209 b 1-6) and as its matter (209 b 6-11) to conclude that Plato maintains that $\chi \dot{\omega} p \alpha$, matter, and space are the same (209 b 11-17).”, we can see the unit $\mathrm{u}$ as the base element of all there is.

With this mindset we can interpret the Planck constant $\mathrm{h}$ as a scaling factor for the base unit, that translates the unit to man made measurement units systems. Furthemore the constants $\mathrm{c}$ and $\mathrm{G}$ (plus the constant k, which is directly dependent on c) can be interpreted as conversion factors from the ideal geometric reality (measured in $\mathrm{u}$ ) to the reality perceived by humans' (as well as other living beings') senses (measured in seconds, meters, kilograms, amperes, kelvins, candelas and/or coulombs, webers, joules etc.).

We can postulate then, that electron is a base excitation of spacetime with two defining properties. One of them being its electromagnetic action at rest (the product of its electric charge and own magnetic flux) e $\cdot 2 \Phi_{0}=u^{2}$ and the other one its excitation activity (the product of its rest mass and excitation/oscillation wavelength, or, in other words, the product of its rest action and the speed of light) $m_{e} \cdot \lambda_{C}=u^{2}$ (in SG rest mass is directly equivalent to rest energy). This description is equivalent to the description derived from P. Dirac's and E. Schrödinger's works by J. Maruani [6]: "the internal structure of the electron should consist of a massless charge describing, at light velocity, a vibrating motion in a domain defined by the Compton wavelength, the measured rest mass being generated by this very internal motion”. 
Since all other charged fermions share the property of electric charge and own magnetic flux quantization with the values of the quanta being equal to the elementary charge and own magnetic flux of electron, it is natural to undertake exploration of the idea, that all of them are different excitations of electromagnetic rest action quantum e $\cdot 2 \Phi_{0}=u^{2}$.

The table below shows, in addition to values of electron properties, hypothetical values of excitation wavelengths and rest masses (energies) of stable quarks, that are in accordance with the current state of knowledge on the subject [7]. There are two more values that characterize the fundamental properties of matter and electromagnetic radiation in the SG, the Planck constant, represented by the unit $\mathrm{u}$ equal to the Planck length $\cdot \sqrt{ }(2 \pi)$ and the Schwinger limit $/ 2 \pi$. The Schwinger limit in the SI is defined by critical values of electric and magnetic fields flux density, beyond which electromagnetic field becomes nonlinear, respectively $\mathrm{E}_{\mathrm{c}}=2 \pi \mathrm{m}_{\mathrm{e}}{ }^{2} \mathrm{c}^{3} /(\mathrm{eh})$ and $\mathrm{B}_{\mathrm{c}}=2 \pi \mathrm{m}_{\mathrm{e}}{ }^{2} \mathrm{c}^{2} /(\mathrm{eh})$, what translates to $V\left(\mathrm{E}_{\mathrm{c}} \mathrm{B}_{\mathrm{c}}\right) /(2 \pi)=\mathrm{m}_{\mathrm{e}}{ }^{2} / \mathrm{e}$ in the $\mathrm{SG}$.

In the $S G$ e $\cdot 2 \Phi_{0}=u^{2}=m_{e} \cdot \lambda_{C}$ and in the SI e $\cdot 2 \Phi_{0}=\mathrm{h}=\mathrm{c} \cdot \mathrm{m}_{\mathrm{e}} \cdot \lambda_{\mathrm{C}}$ and similarly in the $S G \mathrm{~V}\left(\mathrm{E}_{\mathrm{c}} \mathrm{B}_{\mathrm{c}}\right) /(2 \pi)=\mathrm{m}_{\mathrm{e}}{ }^{2} / \mathrm{e}$ and in the $\mathrm{SI}$ $V\left(E_{c} B_{c}\right) /(2 \pi)=c^{5 / 2} m_{e}{ }^{2} / e$. The order of the factor of speed of light in vacuum from the relations in the SI, which is missing from their counterparts in the SG, is apparently related to the order of the factor $v(2 \pi \alpha)$ from the SG formulas for the fundamental properties of matter and radiation shown in Table 2.

Table 2: The fundamental properties of stable matter particles and electromagnetic radiation. ${ }^{1,2}$

${ }^{1}$ The first four columns list exponents for the factors from the title row.

${ }^{2}$ The value of the Schwinger e-m wavelength limit $2 \pi$ in $1 / \sqrt{ }(\mathrm{V} / \mathrm{m} \cdot \mathrm{T})$ amounts to $1 / 1.216363348 \cdot 10^{13}$. Conversion factor to $\mathrm{m}: 1 / \sqrt{ }(\mathrm{V} / \mathrm{m} \cdot \mathrm{T})=\sqrt{ } \mathrm{kc} \mathrm{c}^{3} / \mathrm{G} \mid \mathrm{m}$.

\begin{tabular}{|c|c|c|c|c|c|c|c|}
\hline $2 / 3$ & 2 & $\sqrt{ }(2 \pi \alpha)$ & $1 / \alpha$ & Property & SG formula & SG value $(\mathrm{u})$ & SI value \\
\hline & 16 & 5 & 1 & $\begin{array}{c}\text { Schwinger e-m } \\
\text { wavelength limit· } 2 \pi\end{array}$ & $2^{16} \sqrt{ }(2 \pi \alpha)^{5} / \alpha$ & $4.042756610 \cdot 10^{3}$ & $1.364793043 \cdot 10^{-36} \mathrm{~m}$ \\
\hline & 8 & 3 & 1 & $\lambda_{\mathrm{Ce}}$ & $2^{8} \sqrt{ }(2 \pi \alpha)^{3} / \alpha$ & $3.444232279 \cdot 10^{2}$ & $2.426310239 \cdot 10^{-12} \mathrm{~m}$ \\
\hline-0.5 & 5.5 & 3 & 1 & $\lambda_{\mathrm{Cu}}$ & $\sqrt{3} 2^{5} \sqrt{ }(2 \pi \alpha)^{3} / \alpha$ & $7.456981626 \cdot 10^{1}$ & $5.253115760 \cdot 10^{-13} \mathrm{~m}$ \\
\hline \multirow[t]{4}{*}{0.5} & 5 & 3 & 1 & $\lambda_{\mathrm{Cd}}$ & $\sqrt{2} / 32^{5} \sqrt{ }(2 \pi \alpha)^{3} / \alpha$ & $3.515254850 \cdot 10^{1}$ & $2.476342518 \cdot 10^{-13} \mathrm{~m}$ \\
\hline & & 1 & 1 & $2 \Phi_{0}$ & $\sqrt{ }(2 \pi \alpha) / \alpha$ & $2.934318619 \cdot 10^{1}$ & $4.135667696 \cdot 10^{-15} \mathrm{~Wb}$ \\
\hline & & & & $\mathrm{u}=1_{\mathrm{p}} \sqrt{ }(2 \pi)$ & 1 & 1 & $4.051154841 \cdot 10^{-35} \mathrm{~m}$ \\
\hline & & -1 & -1 & e & $1 /(\sqrt{ }(2 \pi \alpha) / \alpha)$ & $3.407946204 \cdot 10^{-2}$ & $1.602176634 \cdot 10^{-19} \mathrm{C}$ \\
\hline-0.5 & -5 & -3 & -1 & $\mathrm{~m}_{\mathrm{d}}$ & $1 /\left(\sqrt{2} / 32^{5} \sqrt{ }(2 \pi \alpha)^{3} / \alpha\right)$ & $2.844743959 \cdot 10^{-2}$ & $\begin{array}{l}8.925336776 \cdot 10^{-30} \mathrm{~kg} \\
\quad\left(5.007 \mathrm{MeV} / \mathrm{c}^{2}\right)\end{array}$ \\
\hline \multirow[t]{3}{*}{0.5} & 5.5 & -3 & -1 & $\mathrm{~m}_{\mathrm{u}}$ & $1 /\left(\sqrt{3} 2^{5} \sqrt{ }(2 \pi \alpha)^{3} / \alpha\right)$ & $1.341025163 \cdot 10^{-2}$ & $\begin{array}{l}4.207444106 \cdot 10^{-30} \mathrm{~kg} \\
\quad\left(2.360 \mathrm{MeV} / \mathrm{c}^{2}\right)\end{array}$ \\
\hline & -8 & -3 & -1 & $\mathrm{~m}_{\mathrm{e}}$ & $1 /\left(2^{8} \sqrt{ }(2 \pi \alpha)^{3} / \alpha\right)$ & $2.903404645 \cdot 10^{-3}$ & $\begin{array}{l}9.109383702 \cdot 10^{-31} \mathrm{~kg} \\
\left(0.511 \mathrm{MeV} / \mathrm{c}^{2}\right)\end{array}$ \\
\hline & -16 & -5 & -1 & $\begin{array}{l}\text { Schwinger e-m flux } \\
\text { density limit/ }(2 \pi)\end{array}$ & $1 /\left(2^{16} \sqrt{ }(2 \pi \alpha)^{5} / \alpha\right)$ & $2.473559743 \cdot 10^{-4}$ & $\begin{array}{c}1.216363348 \cdot 10^{13}\left[\mathrm{~kg} / \mathrm{C} \cdot \sqrt{ }\left(\mathrm{m} / \mathrm{s}^{3}\right)=\right. \\
=\sqrt{ }(\mathrm{V} / \mathrm{m} \cdot \mathrm{T})]\end{array}$ \\
\hline
\end{tabular}


Also a simple formula for quark $s$ fits the experimental data: $\mathrm{m}_{\mathrm{s}}=1 /\left((1 / 3)^{3} \sqrt{ } 1 / 32^{6} \sqrt{ }(2 \pi \alpha)^{3} / \alpha\right) \mathrm{u}$, equivalent of $95.59 \mathrm{MeV} / \mathrm{c}^{2}$; but since it is an unstable particle, one can expect that its decay rate should be reflected in its $S G$ formula. The same applies to all the remaining (unstable) charged fermions. The relevant equations are yet to be proposed.

In the SG the Schwinger limit of electromagnetic field flux density becomes the limit for the scale of physical events. Expressed as the shortest electromagnetic wave wavelength limit it is - in the SI - ca 12 times smaller than the Planck length.

It is interesting to note the regularity in the following formulas (all giving results in the unit $\mathrm{u}$ ):

\begin{tabular}{|c|c|c|}
\hline$\sqrt{ }\left(\mathrm{E}_{\mathrm{c}} \mathrm{B}_{\mathrm{c}}\right) /(2 \pi)$ & $=1 /\left(2^{16} \sqrt{ }(2 \pi \alpha)^{5} / \alpha\right)$ & the Schwinger limit of electromagnetic flux density/ $(2 \pi)$, \\
\hline $\mathrm{m}_{\mathrm{e}}$ & $=1 /\left(2^{8} \sqrt{ }(2 \pi \alpha)^{3} / \alpha\right)$ & electron rest mass, \\
\hline$e^{2}$ & $=1 /(\sqrt{ }(2 \pi \alpha) / \alpha)$ & elementary charge, \\
\hline $2 \Phi_{0}$ & $=\sqrt{ }(2 \pi \alpha) / \alpha$ & double magnetic flux quantum, \\
\hline$\lambda_{\mathrm{Ce}}$ & $=2^{8} \sqrt{ }(2 \pi \alpha)^{3} / \alpha$ & the Compton wavelength for electron, \\
\hline $2 \pi / \sqrt{ }\left(E_{c} B_{c}\right)$ & $=2^{16} \sqrt{ }(2 \pi \alpha)^{5} / \alpha$ & the Schwinger limit of electromagnetic wavelength $\cdot 2 \pi$. \\
\hline
\end{tabular}

They look like a set of harmonic frequencies of the $\sqrt{ }(2 \pi \alpha)$ 'tone' of the base spacetime excitation. Giving also the earlier mentioned relation of the order of the base tone to the order of the speed of light in vacuum factor in the SI counterparts to the SG relations, one can speculate that the differences in the order of the harmonic frequency of the base spacetime tone effect in differences between observable qualities: mass and charge, charge and magnetic flux, particle and wave, matter and radiation. The higher the density of the electromagnetic flux, the perceivably 'more material' it becomes, but not continuously, only in steps, from one harmonic frequency threshold up to another.

Interestingly, one can also notice that in the SG measured electron magnetic moment $\mu_{\mathrm{e}}$ relates to Bohr magneton $\mu_{\mathrm{B}}$ as follows

$$
\mu_{\mathrm{e}}=-\mu_{\mathrm{B}}\left(1+|\mathrm{e}|^{2}-|\mathrm{e}|^{4}-2^{8}|e|^{6}-2^{12}|e|^{8}-2^{16}|e|^{10}-2^{24}|e|^{12}\right),
$$

which relation is another indication of the existence of higher order harmonic excitations of the base spacetime tone: electron. Each order being of different perceived quality, that subsequently interact with each other.

The sum shown in (1) in the brackets results in the value of (below e is replaced by its SG formula $V(\alpha / 2 \pi)$ )

$$
1+\alpha / 2 \pi-(\alpha / 2 \pi)^{2}-2^{8}(\alpha / 2 \pi)^{3}-2^{12}(\alpha / 2 \pi)^{4}-2^{16}(\alpha / 2 \pi)^{5}-2^{24}(\alpha / 2 \pi)^{6} \approx 0.001159652180 .
$$

The value agrees with $<1$ part per billion accuracy with CODATA 2018 recommended value of electron magnetic moment anomaly $\mathrm{a}_{\mathrm{e}} \approx 0.001159652181$. The difference is 6 times larger than the error allowance estimated by CODATA. The value (2) is purely theoretical, assuming no external influence whatsoever on free electron, while the CODATA 2018 recommended value is based on the most precise scientific measurements, but performed on Earth, under not fully understood influence of the planet itself and its surrounding masses and spacetime irregularities as well as external electromagnetic fields on the experimental results.

Conclusions. - The consistent implementation of the natural geometric unit system (the SG) and interpretation of the formulas for base physical properties written in the system lead to two hypotheses that are directly verifiable experimentally. The hypothesized values of the Newtonian gravitational constant and the electron magnetic moment anomaly are consistent to the convincing degree of accuracy with the most contemporary interpretations of the experimental data. The postulated relations leading to the hypothesized values are built in the SG with use of half integer powers of $2, \pi$ and $\alpha$ only. It seems plausible also, as 
show proposed relations for stable quarks, that adding to the mix only half integer powers of 3, could be enough to describe in the $S G$ all the fundamental properties of stable matter and electromagnetic radiation.

The author dedicates this work to his daughter Kornelia.

The author states that there is no conflict of interests in relation to this article. 


\section{REFERENCES}

[1] KOWALSKI J., Natural geometric unit system and gravitational constant in electron charge to mass ratio, preprint submitted to Foundations of Physics, 2021

[2] Brochure sur le SI, English version 2019 https://www.bipm.org/utils/common/pdf/si-brochure/SI-Brochure-9-EN.pdf

[3] SI brochure EN 8th edition 2006 pp. 109, 128 https://www.bipm.org/utils/common/pdf/si_brochure 8 en.pdf

[4] FRITSCHE J., Aristotle on Space, Form, and Matter("Physics" IV:2, 209 B 17-32), Archiv für Begriffsgeschichte, 48 (2006) 45-63

[5] CODATA 2018 recommended values of the physical constants https://physics.nist.gov/cuu/pdf/wall 2018.pdf

[6] MARUANI J. , The Dirac Electron: Spin, Zitterbewegung, the Compton Wavelength, and the Kinetic Foundation of Rest Mass in Quantum Systems in Chemistry and Physics, Progress in Theoretical Chemistry and Physics 26, by NISHIKAWA K. et al., (Springer Science+Business Media, Dordrecht) 2012, Chapt. 2, pp. 23-46.

[7] TANABASHI M. et al. (Particle Data Group), Phys. Rev. D, 98 (2018) 030001 and 2019 update http://pdg.lbl.gov/2019/tables/rpp2019-sum-quarks.pdf 